\title{
Update and New Directions in Therapeutics for Neurological Complications of HIV Infections
}

\author{
Ronald Ellis $^{1}$ (D) Scott L. Letendre ${ }^{1}$
}

Published online: 6 July 2016

(C) The American Society for Experimental NeuroTherapeutics, Inc. 2016

\begin{abstract}
The pace of therapeutic developments in HIV presents unique challenges to the neurologist caring for patients. Combination antiretroviral therapy (cART) is remarkably effective in suppressing viral replication, preventing, and often even reversing disease progression. Still, not every patient benefits from cART for a variety of reasons, ranging from the cost of therapy and the burden of lifelong daily treatment to side effects and inadequate access to medical care. Treatment failure inevitably leads to disease progression and opportunistic complications. Many of these complications, even those that are treatable, produce permanent neurological disability. With ART, immune recovery itself may paradoxically lead to severe neurological disease; strategies for managing so-called immune reconstitution inflammatory syndrome are beginning to show benefits. Effective cART may nevertheless leave in its wake persistent neurocognitive impairment. Treatments for persistent impairment despite virologic suppression and good immune recovery are being tested but are not yet proven. As we shall see, these treatments target several proposed mechanisms including cerebral small vessel disease, which is highly prevalent in HIV. Most recently, an ambitious initiative has been undertaken to develop interventions to eradicate HIV. This will require elimination of all infectious forms of viral nucleic acid throughout the body. The influence of these interventions on the brain remains to be characterized. Meanwhile, clinical investigators continue to develop antiretroviral treatments that optimize effectiveness, convenience, and tolerability, while minimizing longterm toxicities.
\end{abstract}

Ronald Ellis

roellis@ucsd.edu

1 University of California San Diego, San Diego, CA, USA
Keywords HIV · Immune reconstitution inflammatory syndrome · AIDS · eradication · cerebral small vessel disease · antiretroviral $\cdot$ reservoir

\section{Introduction}

Promising new basic science developments come to bear rapidly on clinical decision-making in HIV neurology. But the pace of these developments makes it difficult for a single paper to review them thoroughly. Fortunately, numerous reviews of key areas have been undertaken, many of them published in publicly available venues. This will be a selective review of some of the more important developments. We will focus on recent therapeutic developments in HIV diseases of the central nervous system, leaving peripheral nerve disease to other settings. For the reader interested in a more comprehensive treatment of individual topics, citations are provided.

By way of introduction, readers are reminded of key terminology and concepts in the field. First, HIV is a retrovirus, with a life cycle and pathophysiology very different from that of viruses classically considered by neurologists interested in virology, such as the herpesviruses. For example, where some herpesviruses maintain latency as viral DNA separate from the host nuclear genome, HIV literally writes itself into the host genome through reverse transcription and integration into cellular DNA. Despites its remarkable successes, combination antiretroviral therapy (cART) has no access to this integrated proviral DNA. Second, from the standpoint of diagnosis and therapeutics, while in the past most neurological attention has been propelled by the severe and complex opportunistic conditions that occur in advanced HIV disease, such as progressive multifocal leukoencephalopathy, the research magnifying glass has in the last decade moved its focus from late to early disease because of effective cART. One of the primary 
motivating factors here is the possibility that neurologic disease, both primary and opportunistic, can be prevented by early treatment initiation. In addition, however, it is believed that early infection may present the best opportunity to limit the extensive seeding of proviral DNA in tissue reservoirs.

\section{HIV Reservoirs and the Cure Agenda}

Antiretroviral therapy (ART) suppresses viral replication in most HIV-infected individuals and drastically reduces morbidity and mortality [39]. But a true cure for HIV infection would eliminate the need for antiretroviral therapy [47]. The benefits for individual patients with HIV would be obvious. But perhaps even more important, from a public health perspective, a true cure applied at the population level would also eliminate new infections by blocking transmission.

The principal barrier to cure is the HIV reservoir, which comprises fully replication competent (infectious) copies of retroviral DNA persisting in multiple sites throughout the body $[9,10,26]$, including the brain. Because ART does not eradicate cells harboring HIV DNA [48], plasma viremia generally rebounds quickly after treatment is interrupted [28]. The HIV reservoir is established very early - within the first days to weeks of infection $[2,40,46]$. Even if the brain is only a minor tissue reservoir site, cure strategies must address it, as any reservoir can serve to re-establish systemic infection.

HIV reservoir size is important because it predicts how quickly virus will rebound after interruption of cART. Proof of concept that reservoir size can be reduced is found in studies of early treatment initiation $[27,36]$. While methods for measuring the size of HIV reservoirs in some sites have been developed, these methods are not technically feasible for brain tissue. The same cellular mechanisms that permit each cell's genetic information to be expressed or repressed also act on HIV retroviral DNA. Thus, interventions that attempt to manipulate HIV DNA are very likely to affect cellular DNA as well. Such interventions are actively being developed, but their impact on the brain is unknown.

\section{Combination ART and Treatment of HIV-Associated Neurocognitive Disorders}

Combination ART has yielded enormous benefits for HIVassociated neurocognitive disorders (HAND), as it has for systemic HIV disease [43], though, as we shall see, there are several important caveats and special situations. As with all neurological syndromes, neurocognitive impairment in HIV deserves a diagnostic evaluation before treatment is initiated. Not all neurocognitive impairment in a patient with HIV is caused by $\mathrm{HIV}$, and cART will not benefit impairment that is pre-existing or due to comorbid conditions. In one large multicenter study, more than a third of impaired individuals had confounding comorbidities other than HIV judged of sufficient cumulative severity to explain their cognitive impairment [25].

To understand the impact of cART on HAND, some key general principles of antiretroviral therapy must be appreciated. First, specific antiretroviral drugs from specific classes must be combined in ways that limit the emergence of drug resistance. New antiretroviral medications and drug classes are introduced to the armamentarium regularly. These new drugs are introduced, in part, to overcome limitations of tolerability, convenience, and side effects of older drugs. Also, new drug classes permit practitioners to circumvent antiretroviral resistance acquired during historical past treatment. The recommended combinations change regularly, but up-to-date recommendations are made publicly available through a variety of venues.

Despite the rapid evolution of cART regimens, one consistent finding is that the patient's first treatment regimen typically yields the most substantial neurocognitive improvement [35]. Subsequent changes to cART regimens, typically made to accommodate virologic failure, antiretroviral drug resistance, side effects, or to reduce regimen complexity, have a lesser impact. The largest neurocognitive benefit of cART is typically seen in the first 6-12 months after initiation [14], though smaller improvements may continue thereafter. Subsequent virologic failure is accompanied by an elevated risk of neurocognitive decline.

Two issues that have generated a great deal of scientific attention are the central nervous system (CNS) penetration of antiretroviral drugs and their efficacy in macrophages [34, 44]. Effectively getting antiretrovirals to the cerebrospinal fluid (CSF) can be difficult for several reasons. There are multiple barriers, including molecular pumps such as pglycoprotein and organic anion transporters that actively transfer protease inhibitors out of the brain extracellular fluid back into the systemic circulation. A randomized, multicenter, controlled trial comparing cART regimens with high versus low expected CNS penetration efficacy in individuals with HAND was performed [19]. Neurocognitive performance improved similarly in both treatment arms. However, the study was not fully powered to determine if there is a role for switching to CNS-targeted therapy.

Inhibition of HIV in infected macrophages requires higher antiretroviral drug concentrations than lymphocytes. Since macrophages are the primary cellular targets of HIV in the brain, inadequate control of HIV in these cells may be needed to treat HAND [4]. Because excellent reviews of CNS penetration and macrophage efficacy recently been published $[15$, $32,50]$, no further consideration of them will be given here.

\section{CSF Viral Escape}

At centers where lumbar punctures are performed, disproportionate elevations in CSF viral load are seen regularly. This 
may be a detectable CSF viral load with undetectable plasma in a patient on stable cART, or a CSF viral load that is 5 to 10 fold higher than plasma. Patients with CSF viral escape can be symptomatic [8] or asymptomatic [17]. Reported symptoms are nonspecific and include headache, neck stiffness, confusion, and incoordination. Neurologic examination findings also are variable and include meningismus, encephalopathy, and ataxia. When carefully looked for in large cohorts undergoing regular lumbar punctures, CSF escape may occur in as many as $10 \%$ of patients at some point during follow-up. In these studies, where lumbar punctures are being done for research rather than clinical purposes, the overwhelming majority of cases of virologically defined CSF escape are intermittent (not sustained) and clinically asymptomatic. Thus, at centers where lumbar punctures are not routinely performed, most CSF viral escape events will be missed.

\section{Neuroprotective Strategies}

For many years clinicians have been interested in discovering whether neuroprotective strategies might benefit cognitive function in HIV. At least a dozen agents with varying mechanisms have been evaluated, such as memantine, nimodipine, and selegiline. Excellent reviews of these studies have been published (e.g., [1, 32]). At this time, no specific treatment is supported by large, high-quality, multicenter, randomized clinical trials. Future directions may include looking at CCR5 antagonists and integrase inhibitors, as well as the use of nanoparticles to more effectively deliver drugs to the brain. As discussed in the following, several emerging therapies target the possible contribution of cerebral small vessel disease (CSVD) to HAND. Future work in neuroprotection must be careful to consider possible neurologic toxicities as well as benefits [11].

\section{Immune Reconstitution Inflammatory Syndrome in the CNS}

\section{Introduction to Immune Reconstitution Inflammatory Syndrome}

The paradox of immune reconstitution inflammatory syndrome (IRIS) is the patient who gets worse even while his or her immune system is recovering. IRIS represents an abnormally exuberant response of the recovering immune system to residual antigens of opportunistic pathogens, both dead and alive. IRIS can occur in virtually any organ, but may be particularly devastating when it occurs in the brain. Prompt recognition of IRIS requires a high index of suspicion. As discussed below, treatment involves eradicating the inciting pathogen and managing the immune response to prevent irreversible neural injury. IRIS is not unique to HIV. For example, neurologists involved in the care of patients with multiple sclerosis recognize the potential for serious neurological deterioration with immune recovery after disease modifying therapies are withdrawn [38]. Similar but more profound immune recovery occurs when powerful combination ART is given to very immune-deficient HIV-positive hosts.

\section{Who is Vulnerable to IRIS?}

The best predictors of developing IRIS are a very low CD4 cell count nadir, presence of an opportunistic infection, and a rapid rate of immune recovery, which may be indirectly related to the drop in viral load. There may be a genetic contribution to risk for IRIS. Some studies show that patients with IRIS have greater number of activated effector memory $\mathrm{CD}^{+} \mathrm{T}$ cells expressing high levels of interferon- $\gamma$ and programmed cell death protein 1 , suggesting that they are primed for an immune response [3, 24, 49].

\section{IRIS Subtypes}

IRIS is often subdivided into 2 categories: simultaneous and unmasked. These are defined in terms of whether the pathogen eliciting the immune response had been identified prior to the neurological worsening. Simultaneous IRIS occurs in patients previously treated for an opportunistic infection who experience paradoxical relapse or worsening of signs and symptoms after initiating combination antiretroviral therapy. In unmasked IRIS (also known as delayed IRIS), patients not previously recognized to have a CNS opportunistic infection (perhaps due to a lack of immunocompetence), experience de novo neurologic deterioration in the context of immune recovery on combination ART.

\section{Pathogens Associated with IRIS}

Common opportunistic infections associated with IRIS are Cryptococcus, Mycobacterium tuberculosis (MTB), and JC virus [41]. IRIS is rare in the setting of cerebral toxoplasmosis and primary CNS lymphoma [32]. At times the clinical picture may suggest CNS IRIS (i.e., focal neurological deterioration in a patient with immune recovery on ART), but no specific pathogen can be identified. In these infrequent cases, IRIS may be attributed to HIV itself. In resource-limited settings, MTB is a particularly important cause of IRIS. MTB IRIS can present later than IRIS with other organisms, often 5 to 10 months after restarting combination antiretroviral therapy. It should always be considered in the patient with clinical deterioration and radiological features such as meningeal enhancement or communicating hydrocephalus after starting combination antiretroviral therapy [13]. Cryptococcal IRIS represents an estimated $10 \%$ to $30 \%$ of all cases of CNS IRIS. It has been reported as soon as 14 days after starting 
combination antiretroviral therapy. It should be considered when there is sterile inflammation of CSF and no yeast found from culture $[6,22]$.

\section{Timing of ART Versus Antifungal Therapy Initiation in Cryptococcal Meningitis}

A recent controlled trial found that 26-week mortality in newly diagnosed cryptococcal meningitis was higher in patients who started combination ART earlier (1-2 weeks) versus later (5 weeks) [7]. This was especially true for those with a paucity of white blood cells in their CSF $\left(<5 / \mathrm{mm}^{3}\right)$, though the rate of clinically diagnosed IRIS did not differ between those with and without pleocytosis.

\section{Treatment of IRIS}

Specific treatment of IRIS is often not needed as spontaneous improvement may occur when the overexuberant immune response gradually abates. However, in a patient who is rapidly clinically deteriorating with no other apparent cause, steroids may be given $[31,37]$. A common regimen uses $1 \mathrm{~g}$ methylprednisolone sodium succinate for 5 days, then a 4- to 6-week taper of oral prednisone. The taper is important because the inflammation of immune reconstitution inflammatory syndrome can rebound if steroids are abruptly withdrawn.

\section{CSVD in HIV}

\section{Neuropathologic Evidence that CSVD is Common in HIV}

In a study of postmortem brains, CSVD was frequent, affecting more than half of those with HIV [45]. CSVD was defined as concentric intramural hyalinization of small arteries or arterioles in white matter. It was frequent even in those who died with virologic suppression on cART. Patients with CSVD at postmortem also were more likely to be neurocognitively impaired during life than those without CSVD. Other antemortem predictors of more frequent and severe CSVD were diabetes mellitus, older age, and exposure to HIV protease inhibitors.

These data are consistent with the view that CSVD contributes to brain injury and HAND. Interventions designed to limit CSVD might benefit neurocognitive function during life. However, to make this possible we must have reliable surrogate markers to identify CSVD during life. This might be possible using noninvasive blood flow measures such as functional magnetic resonance imaging and transcranial Doppler, or biomarkers of vascular damage in CSF. An alternative approach is to treat based on risk factor profile. But, as we discuss below, reliable predictors of CSVD in HIV are not yet known, and cannot be simply generalized from predictors in HIV-uninfected individuals.

\section{Mechanisms of CSVD}

The high prevalence of CSVD in HIV likely results from a combination of persistent inflammation and a propensity to develop the metabolic syndrome (MetS), a combination of abdominal obesity, hypertension, dyslipidemia, and insulin resistance. MetS is highly prevalent in virologically suppressed HIV-seropositive individuals on cART. For example, in a study of $>2500 \mathrm{HIV}$-positive individuals initiating their first cART regimen, $20 \%$ already had MetS, and an additional $27 \%$ developed MetS during 3 years of follow-up [33]. MetS was most common with protease inhibitor-based cART regimens but occurred frequently with other regimens as well.

Systemic inflammation (elevated plasma C-reactive protein and interleukin-6) and immune activation (elevated sCD163 and sCD14) are hallmarks of chronic HIV infection, even when viral replication is well controlled with cART $[16,23$, $29,42]$. Both are expected to upregulate inflammation and activation of microglia in the CNS. Insulin resistance (IR), a component of the MetS, is a frequent consequence of systemic inflammation [20]. IR is especially important in the brain, since normal insulin action is necessary for repairing neuronal injury, promoting neurite outgrowth, and regulating substrate/ energy metabolism [12]. IR is linked to CSVD through endothelial dysfunction [30], which reduces cerebral blood flow, limiting delivery of oxygen and energy substrates, [51, 52]. Thus, reducing inflammation and IR will be important in protecting and restoring neuronal health in the CNS.

\section{Treatments for CSVD in HIV}

If CSVD contributes significantly to HAND in virally suppressed patients as suggested by some reports [21], then eliminating the underlying causes of CSVD might benefit HAND. CSVD also occurs in HIV uninfected individuals, and a rational approach used here is to control risk factors such as diabetes mellitus, hypertension, and dyslipidemia. While such an approach is also prudent in HIV, its effectiveness has not been tested. Factors unique to HIV such as persistent immune activation, inflammation, and small vessel toxicities of antiretroviral drugs might need to be targeted specifically to improve CSVD in HIV. Two potential treatment strategies are being tested in randomized clinical trials: pitavastatin and tesamorelin. Statins are good candidates for CSVD in HIV not only because of their lipid lowering effects, but also because they have anti-inflammatory properties, improve endothelial function and increase nitric oxide bioavailability. Statins enhance dynamic blood flow in cerebral small vessels, potentially benefiting cognitive function. Statins have not yet been tested for efficacy in HAND.

Tesamorelin is already approved to treat abdominal obesity related to antiretroviral therapy. The relevant mechanism here is normalization of growth hormone production. But 
tesamorelin also stimulates increased production of insulinlike growth factor, which is neurotrophic in vitro and in vivo in animal models. In fact, tesamorelin has been used to treat neurocognitive impairment outside of HIV. Thus, Baker et al. administered tesamorelin or placebo subcutaneously in a randomized fashion for 20 weeks to 137 HIV-uninfected older adults. Tesamorelin was statistically significantly superior to placebo both globally and on measures of improvement in executive function in both subgroups [5]. A randomized trial of tesamorelin for HAND is currently underway [18].

Required Author Forms Disclosure forms provided by the authors are available with the online version of this article.

\section{References}

1. Ances, B. M. and D. B. Clifford (2008). HIV-associated neurocognitive disorders and the impact of combination antiretroviral therapies. Curr Neurol Neurosci Rep 8(6): 455-461.

2. Andrade, R. M., F. J. Torriani and R. J. Ellis (2014). Acute HIV infection presenting as fulminant meningoencephalitis with massive CSF viral replication. Neurology: Clinical Practice 4(3): 256259.

3. Antonelli, L. R., Y. Mahnke, J. N. Hodge, et al. (2010). Elevated frequencies of highly activated CD4 + T cells in HIV + patients developing immune reconstitution inflammatory syndrome. Blood 116(19): 3818-3827.

4. Aquaro, S., R. Calio, J. Balzarini, M. C. Bellocchi, E. Garaci and C. F. Perno (2002). Macrophages and HIV infection: therapeutical approaches toward this strategic virus reservoir. Antiviral Res 55(2): 209-225.

5. Baker, L. D., S. M. Barsness, S. Borson, et al. (2012). Effects of growth hormone-releasing hormone on cognitive function in adults with mild cognitive impairment and healthy older adults: results of a controlled trial. Arch Neurol 69(11): 1420-1429.

6. Boelaert, J. R., K. H. Goddeeris, L. J. Vanopdenbosch and J. W. Casselman (2004). Relapsing meningitis caused by persistent cryptococcal antigens and immune reconstitution after the initiation of highly active antiretroviral therapy. Aids 18(8): 1223-1224.

7. Boulware, D. R., D. B. Meya, C. Muzoora, et al. (2014). Timing of antiretroviral therapy after diagnosis of cryptococcal meningitis. $\mathrm{N}$ Engl J Med 370(26): 2487-2498.

8. Canestri, A., F. X. Lescure, S. Jaureguiberry, et al. (2010). Discordance between cerebral spinal fluid and plasma HIV replication in patients with neurological symptoms who are receiving suppressive antiretroviral therapy. Clin Infect Dis 50(5): 773-778.

9. Chun, T. W. (1997). Presence of an inducible HIV-1 latent reservoir during highly active antiretroviral therapy. Proc Natl Acad Sci U S A 94: 13193-13197.

10. Chun, T. W., L. Carruth, D. Finzi, et al. (1997). Quantification of latent tissue reservoirs and total body viral load in HIV-1 infection. Nature 387(6629): 183-188.

11. Clifford, D. B. and B. M. Ances (2013). HIV-associated neurocognitive disorder. Lancet Infect Dis 13(11): 976-986.

12. Craft, S. and G. S. Watson (2004). Insulin and neurodegenerative disease: shared and specific mechanisms. Lancet Neurol 3(3): 169178.

13. Crump, J. A., M. J. Tyrer, S. J. Lloyd-Owen, L. Y. Han, M. C. Lipman and M. A. Johnson (1998). Military tuberculosis with paradoxical expansion of intracranial tuberculomas complicating human immunodeficiency virus infection in a patient receiving highly active antiretroviral therapy. Clin Infect Dis 26(4): 10081009.

14. Cysique, L. A., F. Vaida, S. Letendre, et al. (2009). Dynamics of cognitive change in impaired HIV-positive patients initiating antiretroviral therapy. Neurology 73(5): 342-348.

15. Cysique, L. A., E. K. Waters and B. J. Brew (2011). Central nervous system antiretroviral efficacy in HIV infection: a qualitative and quantitative review and implications for future research. BMC Neurol 11: 148.

16. Deeks, S. G., R. Tracy and D. C. Douek (2013). Systemic effects of inflammation on health during chronic HIV infection. Immunity 39(4): 633-645.

17. Eden, A., D. Fuchs, L. Hagberg, et al. (2010). "HIV-1 viral escape in cerebrospinal fluid of subjects on suppressive antiretroviral treatment." J Infect Dis 202(12): 1819-1825.

18. Ellis, R. (2016). Tesamorelin for Cognition in HIV. Available at: https://clinicaltrials.gov/ct2/show/NCT02572323?term= Ellis+tesamorelin\&rank $=1$.

19. Ellis, R. J., S. Letendre, F. Vaida, et al. (2014). Randomized trial of central nervous system-targeted antiretrovirals for HIV-associated neurocognitive disorder. Clin Infect Dis 58(7): 1015-1022.

20. Feinstein, R., H. Kanety, M. Z. Papa, B. Lunenfeld and A. Karasik (1993). Tumor necrosis factor-alpha suppresses insulin-induced tyrosine phosphorylation of insulin receptor and its substrates. J Biol Chem 268(35): 26055-26058.

21. Foley, J., Ettenhofer, M., Wright M. J., et al. (2010). CH Neurocognitive functioning in HIV-1 infection: effects of cerebrovascular risk factors and age. Clin Neuropsychol 24(2):265-285. doi:10.1080/13854040903482830

22. French, M. A. (2009). HIV/AIDS: immune reconstitution inflammatory syndrome: a reappraisal. Clin Infect Dis 48(1): 101-107.

23. French, M. A., M. S. King, J. M. Tschampa, B. A. da Silva and A. L. Landay (2009). Serum immune activation markers are persistently increased in patients with HIV infection after 6 years of antiretroviral therapy despite suppression of viral replication and reconstitution of CD4+ T cells. J Infect Dis 200(8): 1212-1215.

24. Grabmeier-Pfistershammer, K., P. Steinberger, A. Rieger, J. Leitner and N. Kohrgruber (2011). Identification of PD-1 as a unique marker for failing immune reconstitution in HIV-1-infected patients on treatment. J Acquir Immune Defic Syndr 56(2): 118-124.

25. Heaton, R. K., D. B. Clifford, D. R. Franklin, Jr., et al. (2010). HIVassociated neurocognitive disorders persist in the era of potent antiretroviral therapy: CHARTER Study. Neurology 75(23): 20872096.

26. Ho, Y. C., L. Shan, N. N. Hosmane, et al. (2013). Replicationcompetent noninduced proviruses in the latent reservoir increase barrier to HIV-1 cure. Cell 155(3): 540-551.

27. Hocqueloux, L., V. Avettand-Fenoel, S. Jacquot, et al. (2013). Long-term antiretroviral therapy initiated during primary HIV-1 infection is key to achieving both low HIV reservoirs and normal T cell counts. J Antimicrob Chemother 68(5): 1169-1178.

28. Hoen, B., I. Fournier, C. Lacabaratz, et al. (2005). Structured treatment interruptions in primary HIV-1 infection: the ANRS 100 PRIMSTOP trial. J Acquir Immune Defic Syndr 40(3): 307-316.

29. Hunt, P. W., J. N. Martin, E. Sinclair, et al. (2003). T cell activation is associated with lower CD4+ T cell gains in human immunodeficiency virus-infected patients with sustained viral suppression during antiretroviral therapy. J Infect Dis 187(10): 1534-1543.

30. Kim, J. A., M. Montagnani, K. K. Koh and M. J. Quon (2006). Reciprocal relationships between insulin resistance and endothelial dysfunction: molecular and pathophysiological mechanisms. Circulation 113(15): 1888-1904.

31. Kranick, S. M., P. H. Goncalves, M. Stetler-Stevenson, et al. (2015). Paradoxical central nervous system immune reconstitution syndrome in acquired immunodeficiency syndrome-related primary 
central nervous system lymphoma. Haematologica 100(1): e21e24.

32. Kranick, S. M. and A. Nath (2012). Neurologic complications of HIV-1 infection and its treatment in the era of antiretroviral therapy. Continuum (Minneap Minn) 18: 1319-1337.

33. Krishnan, S., J. T. Schouten, B. Atkinson, et al. (2015). Changes in metabolic syndrome status after initiation of antiretroviral therapy. $\mathrm{J}$ Acquir Immune Defic Syndr 68(1): 73-80.

34. Kumar, A. and G. Herbein (2014). The macrophage: a therapeutic target in HIV-1 infection. Mol Cell Ther 2(1): 1-15.

35. Letendre, S. L., J. A. McCutchan, M. E. Childers, et al. (2004). Enhancing antiretroviral therapy for human immunodeficiency virus cognitive disorders. Ann Neurol 56(3): 416-423.

36. Li, J. Z., B. Etemad, H. Ahmed, et al. (2016). The size of the expressed HIV reservoir predicts timing of viral rebound after treatment interruption. Aids 30(3): 343-353.

37. McComsey, G. A., C. C. Whalen, S. D. Mawhorter, et al. (2001). Placebo-controlled trial of prednisone in advanced HIV-1 infection. Aids 15(3): 321-327.

38. Miravalle, A., R. Jensen and R. P. Kinkel (2011). Immune reconstitution inflammatory syndrome in patients with multiple sclerosis following cessation of natalizumab therapy. Arch Neurol 68(2): 186-191.

39. Palella, F. J., Jr., R. K. Baker, A. C. Moorman, et al. (2006). "Mortality in the highly active antiretroviral therapy era: changing causes of death and disease in the HIV outpatient study." J Acquir Immune Defic Syndr 43(1): 27-34.

40. Pilcher, C. D., D. C. Shugars, S. A. Fiscus, et al. (2001). HIV in body fluids during primary HIV infection: implications for pathogenesis, treatment and public health. Aids 15(7): 837-845.

41. Post, M. J., M. M. Thurnher, D. B. Clifford, et al. (2013). CNSimmune reconstitution inflammatory syndrome in the setting of HIV infection, part 1: overview and discussion of progressive multifocal leukoencephalopathy-immune reconstitution inflammatory syndrome and cryptococcal-immune reconstitution inflammatory syndrome. AJNR Am J Neuroradiol 34(7): 1297-1307.

42. Rajasuriar, R., G. Khoury, A. Kamarulzaman, M. A. French, P. U. Cameron and S. R. Lewin (2013). Persistent immune activation in chronic HIV infection: do any interventions work? AIDS 27(8): 1199-1208.

43. Schouten, J., P. Cinque, M. Gisslen, P. Reiss and P. Portegies (2011). HIV-1 infection and cognitive impairment in the cART era: a review. Aids 25(5): 561-575.

44. Shikuma, C. M., B. Nakamoto, B. Shiramizu, et al. (2012). Antiretroviral monocyte efficacy score linked to cognitive impairment in HIV. Antivir Ther 17(7): 1233-1242.

45. Soontornniyomkij, V., A. Umlauf, S. A. Chung, et al. (2014). HIV protease inhibitor exposure predicts cerebral small vessel disease. AIDS 28(9): 1297-1306.

46. Spudich, S. (2013). HIV and neurocognitive dysfunction. Curr HIV/AIDS Rep 10(3): 235-243.

47. Thornhill, J., S. Fidler and J. Frater (2015). Advancing the HIV cure agenda: the next 5 years. Curr Opin Infect Dis 28(1): 1-9.

48. Wong, J. K., M. Hezareh, H. F. Gunthard, et al. (1997). Recovery of replication-competent HIV despite prolonged suppression of plasma viremia. Science 278(5341): 1291-1295.

49. Worsley, C. M., M. S. Suchard, W. S. Stevens, A. Van Rie and D. M. Murdoch (2010). Multi-analyte profiling of ten cytokines in South African HIV-infected patients with Immune Reconstitution Inflammatory Syndrome (IRIS). AIDS Res Ther 7: 36-36.

50. Yilmaz, A. and M. Gisslen (2014). Treatment of HIV in the central nervous system. Semin Neurol 34(1): 14-20.

51. Zlokovic, B. V. (2008). The blood-brain barrier in health and chronic neurodegenerative disorders. Neuron 57(2): 178-201.

52. Zlokovic, B. V. (2011). Neurovascular pathways to neurodegeneration in Alzheimer's disease and other disorders. Nat Rev Neurosci 12(12): 723-738. 